\title{
Respuesta del germoplasma de las especies diploides tuberosas de Solanum a la inducción de callos para la manipulación de ploidia
}

\author{
Matilde Orrillo*; Kazuo Watanabe**
}

\section{RESUMEN}

Materiales genéticos de Solanum diploides, de varios orígenes, incluyendo especies silvestres, especies cultivadas, haploides provenientes de variedades tetraploides e híbridos entre los mencionados grupos, fueron empleados para duplicar cromosomas in vitro. Para esto se uso ribósido de zeatina, como una parte del programa de mejoramiento de la papa con germoplasma silvestre de Solanum. Para la inducción de callos y la subsiguiente regeneración de brotes en medio sólido, se usaron entrenudos de tallo y segmentos de hoja de plántulas in vitro. Los entrenudos de tallo respondieron mejor que los segmentos de hoja. Los genotipos híbridos reaccionaron bien en el medio y regeneraron brotes. Los haploides de variedades tetraploides también mostraron alguna respuesta en la formación de callos y en la regeneración de brotes, independientemente de su nivel de endocría. Por otro lado, los genotipos de especies silvestres y sus progenies no mostraron una inducción vigorosa de callos ni regeneración de brotes. La modificación del medio, cambiando las concentraciones de hormonas y de sacarosa no mejoró significativamente la inducción de callos en las especies silvestres y en sus progenies. Parece ser que una amplia variación de alelos es importante para una buena inducción de callos y regeneración de brotes, más que el nivel de endocría. Más aún, los resultados sugirieron que algunos factores hereditarios están involucrados en la habilidad para crecer en cultivo de tejidos, debido a que esta habilidad de genotipos parentales particulares afectó a sus progenies.

Palabras claves adicionales: duplicación cromosómica, mejoramiento de germoplasma, especies tuberosas de Solanum, ribósido de zeatína.

Aceptado para publicación: mayo 9, 1996

\footnotetext{
* Citogenetista, Departamento de Recursos Genéticos, Centro Internacional de la Papa, Apartado 1558, Lima, Perú.

** Citogenetista asociado, Associate Professor, Department of Biotechnology Science, Faculty of Bioscience and Technology, Kinki University. 930 Nishimitani, Uchita-Cho, Naka-Gunn, Wakayama, 649-64, Japan.
} 


\title{
SUMMARY
}

\section{Response of Diploid Tuber-Bearing Solanum Species to Callus Induction for Ploidy Manipulation}

\begin{abstract}
Diploid tuber-bearing Solanum from various sources including wild species, cultivated species, haploids from tetraploid varieties, and hybrids among the above groups were employed for in vitro chromosome doubling by the use of zeatin riboside, as a part of a germplasm enhancement program with wild Solanums to improve the potato crop. Stem internodes and leaf segments from in vitro plantlets were used for callus induction, and subsequent shoot regeneration in solid medium. Stem internodes generally gave better response than leaf segments. Hybrid genotypes reacted well in the medium and regenerated shoots. Haploids from tetraploid varieties also exhibited some response in callus formation and shoot regeneration regardless of their level of inbreeding. On the other hand, genotypes of wild species and their progenies did not show vigorous callus induction and shoot regeneration. Modification of the medium by changing the concentrations of hormones and sucrose did not yield significantly better callus induction in wild species and their progenies. It appears that wide allelic variation rather than level of inbreeding is important to have a good callus induction and shoot regeneration. Further, it seemed provisionally that some hereditary factors are involved in the tissue culture ability since tissue culture ability of particular parenteral genotypes influenced the progeny performance.
\end{abstract}

Additional index words: cromosomic doubling, germplasm enhancement, tuber-bearing Solanum species, zeatin riboside.

\section{INTRODUCCIÓN}

El mejoramiento de papa a nivel diploide es una herramienta útil para la introgresión de genes valiosos del germoplasma silvestres y/o exótico a la papa tetraploide $(11,15)$. Los atributos genéticos valiosos en papas diploides pueden ser trasmitidos al nivel tetraploide por cuatro caminos principales (14,23): 1) fusionamiento de gametos 2n, 2) tratamiento con colchicina, 3) duplicación del número cromosómico in vitro por agentes como el ribósido de zeatina y el benzilamino purina (BAP), y 4) fusión celular. Las ventajas genéticas de los gametos $2 \mathrm{n}$ sobre otros métodos y técnicas han sido propuestas $(14,15,23)$. 
Cuando no se encuentran gametos 2n en papas diploides de interés, cuando los efectos citoplasmáticos son importantes para la expresión de características útiles, o cuando se presenta baja fertilidad masculina o femenina, se debe considerar el uso de otros métodos. El tratamiento con colchicina a menudo produce quimeras y la eficiencia es baja $(4,7,20)$. Las técnicas de fusión celular todavía requieren mejoramiento en la eficiencia de la regeneración, y en el control de la estabilidad genética $(8,12,25)$.

El trabajo pionero in vitro sobre manipulación de ploidía por inducción de vástagos adventicios con el uso de BAP o ribósido de zeatina demostró un potencial para introducir características genéticas de papas diploides a tetraploides $(9,18,19,23)$. La mayor ventaja de esta técnica sobre el tratamiento con colchicina es una alta eficiencia de duplicación y una presencia no significativa de quimeras en los regenerantes. Sin embargo, hasta ahora la técnica ha sido principalmente probada en híbridos interespecíficos altamente heterocigóticos, y no ha sido experimentada en otras fuentes de germoplasma. No se tiene conocimiento sobre la utilidad de esta técnica usando diversas fuentes de origen genético. Se sabe que especies silvestres tienen generalmente una baja eficiencia para el cultivo de tejidos, tales como el cultivo de anteras $(10,21)$, por lo que para duplicación in vitro de especies silvestres se puede requerir de procedimientos especiales. También la endocría en haploides derivados de variedades tetraploides puede tener un efecto sobre la formación de brotes adventicios.

Esta investigación fue hecha para medir la respuesta a la manipulación de ploidía in vitro del germoplasma diploide. Especies silvestres, haploides de variedades tetraploides, y sus híbridos fueron incluidos en este estudio.

\section{MATERIALES Y MÉTODOS}

Los antecedentes genéticos de los genotipos diploides usados en este experimento y sus correspondientes progenitores están indicados en la Tabla 1.

Las familias B11A, B11B, M-200 fueron gentilmente proporcionadas por la Dra. M.W. Bonierbale, Departamento de Mejoramiento de Plantas, Universidad de Cornell, USA y, las HHI-9 y USW2230 fueron proporcionadas por el Dr. R. E. Hanneman, Jr., Departamento de Horticultura, Universidad de Wisconsin-Madison y USDA_ARS, USA por intermedio de la Dra. Bonierbale. 
Tabla 1. Origen genético de genotipos de papas diploides usados para duplicación de cromosomas in vitro y de sus progenitores, con respecto a las especies involucradas.

\begin{tabular}{|c|c|c|}
\hline Genotipo & Categoría & $\begin{array}{l}\text { Pedigree/ especies } \\
\text { involucradas }\end{array}$ \\
\hline${\mathrm{B} 11 \mathrm{~A}^{\mathrm{b}}}$ & silvestre & ber PI 473331 \\
\hline $\mathrm{B} 11 \mathrm{~B}^{\mathrm{b}}$ & silvestre & ber PI 473331 \\
\hline CIP760147.7 & silvestre & Spl PI 230502 \\
\hline HHI- $9^{c}$ & haploide & tbr, adg, chc? \\
\hline USW2230 ${ }^{c}$ & haploide & tbr, adg, chc? \\
\hline$(2 x T S-2)_{5}$ & haploide & tbr, adg, chc? \\
\hline$(2 X 377964.5)_{5}$ & haploide & tbr, adg, chc \\
\hline$(2 x V-2)_{7}$ & haploide & tbr, adg \\
\hline$(2 x L T-9)_{7}$ & haploide & tbr, adg \\
\hline CCC 1386.26 & híbrido & phu inter cross \\
\hline M200 b & híbrido & USW2230xPI473331 \\
\hline MI 49.10 & híbrido & adg,chc,phu-stn,spl,tbr \\
\hline PTM 1.33 & híbrido & adg,chc,phu,spl ${ }^{\mathrm{d}}$,tbr \\
\hline РTM 15.22 & híbrido & adg,chc,phu,spl ${ }^{\mathrm{d}}$,tbr \\
\hline РTM17.27 & híbrido & adg,chc,phu,spl ${ }^{\mathrm{d}}$,tbr \\
\hline$\{(2 \times 377964.5), x \text { PTM } 1.33\}_{3}$ & híbrido & adg,chc,phu,spl ,tbr \\
\hline$\{(2 \times 377964.5), x \text { PTM } 15.22\}_{23}$ & híbrido & adg,chc,phu,spl ${ }^{\mathrm{d}}$,tbr \\
\hline$\left(\mathrm{PTM} 1.33 \times\right.$ PTM15.22) ${ }_{4}$ & híbrido & adg,chc,phu,spl ,tbr \\
\hline$($ PTM 1.33 x РТМ 17.27) & híbrido & adg,chc,phu,spl ${ }^{\mathrm{d}}$,tbr \\
\hline$(\mathrm{PTM} 1.33$ x CIP760147.7) & híbrido & adg,chc,phu,spl d,tbr \\
\hline$(\mathrm{PTM} 1.33$ x OP760147.7) & híbrido & adg,chc,phu,spl d,tbr \\
\hline$\left\{(2 \mathrm{x} \text { TS-2), } \mathrm{x} \text { PTM } 1.33\}_{7}\right.$ & híbrido & adg,chc,phu,spl d,tbr \\
\hline$\{(2 \mathrm{xTS}-2), \mathrm{xPTM} 1.33\})\}_{18}$ & híbrido & adg,chc,phu,spl d,tbr \\
\hline$\left.\left.\left\{(2 \times T S-2)_{5} \times P T M 1.33\right\}\right)\right\}_{24}$ & híbrido & adg,chc,phu,spl d,tbr \\
\hline$\{(2 \mathrm{x}$ TS-2), $\mathrm{x}$ PTM 1.33 $\})\}_{29}$ & híbrido & adg,chc,phu,spl ${ }^{\mathrm{d}}$,tbr \\
\hline
\end{tabular}

a Abreviaturas para Solanum berthaultii (ber), S. sparsipilum (spl), S. tuberosum ssp. tuberosum (tbr), S. tuberosum ssp. andigena (adg), S. chacoense (che), S. phureja (phu), S. stenotomun (stn), respectivamente.

b Suministrado por M. W. Bonierbale, Dept. de Mejoramiento de Plantas, Universidad de Cornell, Estados Unidos.

c Proporcionado por R.E. Hanneman, Jr., Dept. Horticultura, Universidad de. Wisconsin, Madison, Estados Unidos, por intermedio de la Dra. Bonierbale.

d $\quad$ CIP 760147.7

Los procedimientos usados en este experimento se basaron en Sonnino y col. ${ }^{(23)}$ 
Se usaron dos tipos de explantes de plantas in vitro: 1) segmentos de entrenudos de tallos, de $5 \mathrm{~mm}$ a $10 \mathrm{~mm}$ de longitud los cuales fueron cortados longitudinalmente, y 2) segmentos de hojas con vena media. Estos explantes fueron cultivados en placas de Petri conteniendo medio de cultivo sólido: Murashige Minimal Organic Medium (Laboratorio Gibco), con 5\% w/v de sacarosa y $0.8 \%$ w/v de agar, $3 \mathrm{mg} \mathrm{l}^{-1}$ ribósido de zeatina, $2 \mathrm{mg} \mathrm{l}^{-1}$ ácido indol acético (IAA), y $10 \mathrm{mg} \mathrm{I}^{-1}$ de ácido giberélico $\left(\mathrm{GA}_{3}\right)$, a pH 5.8. Después del autoclavado se distribuyeron $23 \mathrm{~cm}^{3}$ de medio por placa petri $(100 \mathrm{~mm}$ $\mathrm{x} 15 \mathrm{~mm})$. Los explantes fueron mantenidos en el medio por tres semanas, y fueron subcultivados en el mismo medio hasta tres veces.

La incubación se hizo a $20^{\circ} \mathrm{C}$ bajo 3000 lux de intensidad con lámpara Daylight Gro-lux por 16 horas luz día.

Los brotes de callo se propagaron en medio MSA (CIP): Murashige Minimal Organic Medium, más $10 \mathrm{ml}$ de solución base $\left(0.1 \mathrm{mgl}^{-1}\right.$ de ácido giberélico, $2.0 \mathrm{mg}^{1}{ }^{-1}$ de glicina, $0.5 \mathrm{mg} \mathrm{l}^{-1}$ de ácido nicotínico, $0.5 \mathrm{mg} \mathrm{l}^{-1}$ de piridoxina HCL, $0.4 \mathrm{mg} \mathrm{l}^{-1}$ de Tiamina HCL, sacarosa 2.5\%, y $0.4 \%$ de Phytagel). Estos brotes se incubaron usando la misma condición descrita para la inducción de callos.

El nivel de ploidía se determinó en los brotes regenerados, los cuales originalmente provenían de segmentos internodales. Se contó el número de cloroplastos en células oclusivas para determinar el efecto de manipulación de ploidía en la capa L1, y el número cromosómico en las raíces para confirmar el efecto en la capa L3 $(4,9,23)$.

\section{RESULTADOS Y DISCUSIÓN}

La eficiencia de inducción de callos y la regeneración de brotes adventicios fue mejor en segmentos internodales de tallo que en segmentos de hojas (Tabla 2). La prueba del Chi-cuadrado para homogeneidad indicó una diferencia significativa para la regeneración entre entrenudo de tallos y hojas $(0.025<\mathrm{p}<0.05)$. El uso de órganos in vitro o in vivo puede influenciar el resultado. Roest y Bokelmann ${ }^{(18,19)}$ informaron que segmentos de hoja provenientes de invernadero tuvieron una buena respuesta, en contraste con los de origen in vitro del presente experimento. Sin embargo, los brotes adventicios pueden ser obtenidos de segmentos de hoja, segmentos de tallo, callos y raíces $(5,18,19,23)$.

Nuestros resultados parecen indicar que los entrenudos de tallo (in vitro) son adecuados para duplicación de cromosomas (in vitro). En la mayoría de los casos los genotipos con habilidad para la formación para callos (in vitro), pueden ser propagados rápida y fácilmente. 
Tabla 2. Respuesta de genotipos diploides a la inducción de callos y regeneración de brotes

\begin{tabular}{|c|c|c|c|c|c|c|}
\hline \multirow[b]{2}{*}{ Genotipo } & \multicolumn{2}{|c|}{$\begin{array}{c}\mathrm{N}^{\circ} \text { de segmentos } \\
\text { usados }\end{array}$} & \multicolumn{2}{|c|}{$\begin{array}{c}\mathrm{N}^{\circ} \text { de callos } \\
\text { formados (\%) }\end{array}$} & \multicolumn{2}{|c|}{$\begin{array}{c}\mathrm{N}^{\circ} \text { de brotes por } \\
\text { callo }\end{array}$} \\
\hline & Tallo & hoja & tallo & hoja & tallo & hoja \\
\hline B11A & 20 & 20 & 0 & 0 & 0 & 0 \\
\hline B11B & 20 & 20 & 0 & 0 & 0 & 0 \\
\hline CIP 760147.7 & 140 & 140 & 0 & 0 & 0 & 0 \\
\hline CCC 1386.26 & 100 & 100 & 24 & 10 & $1.125^{\mathrm{ab}}$ & 11.3 \\
\hline Hffl-9 & 20 & 12 & 60 & 0 & $5.8^{\mathrm{ab}}$ & 0 \\
\hline US W 2230 & 16 & 12 & 18.8 & 0 & $11.0^{\mathrm{ab}}$ & 0 \\
\hline$(2 x T S-2)_{5}$ & 20 & 20 & 15 & 5 & 3.3 & 2.5 \\
\hline$(2 X 377964.5)_{5}$ & 20 & 20 & 35 & 10 & $2.2^{\mathrm{ab}}$ & 4.0 \\
\hline$(2 x V-2)_{7}$ & 25 & 25 & 8 & 4 & $5.0^{\mathrm{a}}$ & 0 \\
\hline$(2 x L T-9)_{7}$ & 30 & 30 & 13.3 & 6.7 & $3.8^{\mathrm{a}}$ & 0.5 \\
\hline M200-28 & 20 & 20 & 75 & 0 & $1.3^{\mathrm{ab}}$ & 0 \\
\hline M200-32 & 20 & 20 & 90 & 0 & $1.8^{\mathrm{ab}}$ & 0 \\
\hline M200-38 & 20 & 20 & 75 & 0 & $1.3^{\mathrm{ab}}$ & 0 \\
\hline MI 49.10 & 76 & 60 & 26.3 & 25 & $13 .^{a b}$ & 15.3 \\
\hline$\left\{(2 \times 377964.5)_{5} \times \mathrm{PTM} 1.33\right\}_{3}$ & 30 & 0 & 6.6 & 0 & $2.1^{\mathrm{a}}$ & 0 \\
\hline$\left\{(2 \times 377964.5)_{5} \times \mathrm{PTM} 15.22\right\}_{23}$ & 30 & 0 & 6.6 & 0 & 0 & 0 \\
\hline$\left(\right.$ PTM1.33xPTM15.22) ${ }_{4}$ & 20 & 0 & 0 & 0 & 0 & 0 \\
\hline$(\mathrm{PTM} 1.33 \times \mathrm{PTM} 17.27)_{17}$ & 20 & 0 & 5 & 0 & $1.8^{\mathrm{a}}$ & 0 \\
\hline$(\text { PTM1. } 33 \text { x CIP 760147.7) })_{1}$ & 20 & 0 & 0 & 0 & 0 & 0 \\
\hline$(\text { PTM1.33xCIP760147.7) })_{19}$ & 20 & 0 & 0 & 0 & 0 & 0 \\
\hline$\{(2 \mathrm{xTS}-2) 5 \times \mathrm{XTM} 1.33\}_{7}$ & 120 & 48 & 4.2 & 0 & $0.6^{\mathrm{a}}$ & 0 \\
\hline$\{(2 x T S-2) 5 x P T M 1.33\})\}_{18}$ & 68 & 76 & 8.8 & 0 & $6.0^{\mathrm{a}}$ & 0 \\
\hline$\{(2 x T S-2) 5 x P T M 1.33\})\}_{24}$ & 60 & 88 & 5 & 1.1 & $0.5^{\mathrm{a}}$ & 0 \\
\hline$\{(2 \times T S-2) 5 x P T M 1.33\})\}_{29}$ & 90 & 50 & 3.3 & 01 & $4.3^{\mathrm{a}}$ & 0 \\
\hline
\end{tabular}

a Ploidía determinada por contaje del número de cloroplastos en células guardia y por contaje del número cromosómico en raíces.

b Por lo menos una planta tetraploide fue identificada.

La prueba Chi-cuadrado para homogeneidad sobre la inducción de callos entre nudo de tallos y segmentos de hojas fue significativa $\mathrm{x}^{2}=37.3307, \mathrm{df}=23$, $0.025<p<0.05$.

Ninguno de los tres genotipos de especies silvestres usados produjeron buenos callos o brotes adventicios (Tabla 2). Sin embargo, a pesar de que se usaron unos pocos genotipos en este experimento, parece que la habilidad de estas especies silvestres para crecer en medio de cultivo, es generalmente baja. Previamente se ha informado que la habilidad para cultivo de anteras de especies silvestres es generalmente baja $(2,10,21,22)$. 
Se hizo un intento para mejorar la respuesta de genotipos de especies silvestres por modificación de la concentración de hormonas y sacarosa (17), y por el uso de diferentes tipos de órganos (in vitro) e (in vivo), pero no se obtuvo regeneración. Es necesario investigar más para mejorar el cultivo de tejidos de las especies silvestres.

Los haploides de variedades tetraploides demostraron buena respuesta a la inducción de callos y regeneración (Tabla 2), a pesar del nivel de endocría de esos haploides. Los haploides usualmente tienen un alto nivel de endocría (13), generalmente asociada a una depresión por endogamia para vigor general en plantas haploides.

Aparentemente, el nivel de endocría no afecta significativamente el potencial de inducción de callos y regeneración de brotes en los haploides utilizados en el experimento. Además, parece que una amplia variación alélica en haploides, debido a su pedigree o herencia genealógica más que al nivel de endocría, podría ser importante para una buena inducción de callos y regeneración de brotes, ya que las especies silvestres son exógamas, con menor endocría. Las especies silvestres con menor diversidad alélica, no dan respuesta (Tablas 1 y 2).

Los híbridos altamente heterocigotos produjeron dos categorías (Tabla 2): 1) un nivel relativamente bueno de inducción de callos seguido por regenerantes tetraploides en las familias CCC1386.26, MI 49.10 y M-200, y 2) un bajo nivel de inducción de callos (menos del 10\% de los segmentos) en híbridos con participación activa de CIP 760147.7 (Tabla 1). Aunque es difícil estimar en estos híbridos el porcentaje de participación del CIP 760147.7, debido al origen en polinizaciones mixtas al empezar la generación de los progenitores, parece ser que usando este clon, se obtuvo en estos híbridos menor respuesta. De esos resultados, se deduce que puede existir algún control hereditario para la inducción de callos y habilidad de regeneración, puesto que pedigrees con CIP 76014.7 dieron bajos niveles de respuesta (Tabla 1). Además, este efecto inhibitorio de CIP 760147.7 parece ser parcialmente heredado ya que es expresado en aproximadamente igual proporción a la contribución de contenido genético de este clon al genoma de los clones evaluados. El control genético de la inducción de callos y la regeneración ha sido reportado en cultivo de anteras de papa y sus parientes $(2,22,24)$, y en muchos cereales $(1,6,16)$. Las observaciones reportadas aquí proveen evidencia adicional de la herencia en la inducción de callos y regeneración.

Se determinó la ploidía en L1 y L3 (Tabla 2) de los regenerantes de entrenudos de tallo. No se observaron quimeras desde que el promedio del número de cloroplastos por célula oclusivas fue consistente con el nivel de ploidía en las raíces. Esto es una confirmación adicional de reportes previos $(3,9,18,19,23)$. Se identificaron regenerantes tetraploides a partir de varios genotipos. Parece ser que una vez producida la inducción exitosa de callos, la probabilidad de obtener regenerantes tetraploides sería alta. 


\section{AGRADECIMIENTOS}

Estamos muy agradecidos a los Drs. N. W. Bonierbale y R.L.Plaisted por su lectura crítica del manuscrito.

\section{REFERENCIAS BIBLIOGRÁFICAS}

1. Agache, S.; De Buyser, J.; Henry, Y; Snape, J.W. 1988. Studies of the genetic relationship between another culture and somatic tissue culture abilities in wheat. PlantBreed.100:26-33.

2. Cappadocia M, D.; Cheng.S. K.; Ludlum- Simonette.R. 1984. Plant regeneration from in vitro culture of anthers of Solanum chacoense Bitt. and interespecific diploid hybrids S. tuberosum L. x S. chacoense Bitt. Theor. Appl.Genet. 69:139-143

3. Cardi, T.; Carputo, D.; Frusciante, L. 1992. In vitro shoot regeneration and chromosome doubling in 2x an 3x Potato clones. Am. Potato J. 69:1-12.

4. De, Maine, M.J.; Fantes. J. A. 1983. The results of colchicine treatment of dihaploids and their implications regarding efficiency of chromosome doubling and potato histogeny. Potato Res. 26: 289-294.

5. Espinoza, N.O.; Dodds, J. H. 1985. Adventitious shoot formation on cultured potato roots. Plant Sci. 41: 121-124.

6. Foroughi-Wehr, B.; Friedt, W.; Wenzel. G. 1982. On the genetic improvement of androgenetic haploid formation in Hordeum vulgare L. Theor. Appl. Genet. 62:233-239.

7. Frandsen, N.0.1967. Ploidy chimeras from haploid potato clones. Zeitschrifl fur Pflanzenzuchlung 57:123-145.

8. Helgeson, J.P.; Haberlach, G. T; Pohlman, J.; Austin, S. 1988. Somatic fusion of Solanum species. Plant Cell Tissue Organ Culture 12:185-187.

9. Hermsen, J. G. Th.; Ramanna, M. S.; Roest, S.; Bokelmann S. \& G. S. 1981. Chromosome doubling through adventitious shoot formation on in vitro cultivated leaf explants from diploid interespecific potato hybrids. Euphytica 30:239-246. 
10. Irikura, Y. 1975. Induction of haploids plants by anther culture in tuberbearing species and interespecific hybrids of Solanum. Potato Res. 18: 133140.

11. Iwanaga, M. 1984. Potato breeding for combined resistance lo tropical pests by the ploidy level manipulation approach: progress in extraction of haploids with male fertility and $2 \mathrm{n}$ pollen. Symp. Intl. Soc. Trop. Root. Crops, International Potato Center, Lima, Perú, 21-126, Feb., 1983.

12. Lenzini, Z.; Earle, E. D.; Plaisled, R. L. 1990. Insect resistant plants with improved horticultural traits from interespecific potato hybrids grown in vitro. Theor. Appl. Genet. 80:95-104.

13. Mendiburo, A. O.; Peloquin, S. J.; Mok. D. W. S. 1974. Potato breeding with haploids and $2 \mathrm{n}$ gametes . In K Kasha (ed.). Haploids in higher plants., p 249-258.

14. Mendiburo, A. O.; Peloquin, S. J. 1977. Bilateral sexual polyploidization in Potatoes. Euphytica 26:573-583.

15. Peloquin, S. J.; Yerk, G. L.; Werner, J. E.; Darmo. L. 1989. Potato breeding with haploids and 2n gametes. Genome 31:1000-1004.

16. Petolino, J. F.; Thompson, S. A. 1987. Genetic analysis of anther culture response in maize. Theor. Appl. Genet. 74:284-286.

17. Pijnacker, L. P; Ferwerda, M. A 1990. Effect of sucrose on polyploidizalion in early callus cultures of Solanum tuberosum. Plant Cell, Tissue and Organ Culture 21:153-157.

18. Roest, S.; Bokelmann, G. S. 1976. Vegetative propagation of Solanum tuberosum L. in vitro. Potato Res. 19:173-178.

19. Roest, S.; Bokelmann, G.S. 1980. In vitro adventitious bud techniques for vegetative propagation and mutation breeding of potato (Solanum tuberosum L.) 1. Vegetative propagation in vitro through adventitous shoot formation. Potato Res. 23:167-181.

20. Ross, R.; Dionne, L. A.; Hougas, R. W. 1967. Doubling the Chromosome number of selected Solanum genotypes Eur. Potato J. 10:37-52.

21. Simon, P. W.; Peloquin, S. J. 1977. The influence of paternal species on the origin of callus in anther culture of Solanum hybrids. Theor. Appl. Genet. 50:53-56. 
22. Singsit, C; Veilleux, R.E. 1989. Intra- and interespecific transmission of androgenetic competence in diploid Potato species. Euphytica 43:105-112.

23. Sonnino,A.;Iwanaga,M.;Hinostroza,G. 1988.Chromosome number doubling of 2x Potato lines with diverse genetic background through tissue culture. Potato Res. 31:627-631.

24. Uhrig, H. 1985. Genetic selection and liquid medium conditions improve the yield of androgenetic plants from diploid Potatoes. Theor. App. Genet. 71:455-460.

25. Wenzel, G.; Schieder, O.; Prezewozny, T.; Sopory, S. K.; Melchers, G. 1979. Comparison of single cell culture derived Solanum tuberosum L. plants and model for their application in breeding programs. Theor. App. Genet. 55:49-55. 